\title{
NOTE ON THE HOMOLOGY OF A FIBER PRODUCT OF GROUPS
}

\section{G. S. RINEHART}

Abstract. Spectral sequences are derived for the homology and cohomology of a fiber product of groups with coefficients in a tensor product module. These generalize the Hochschild-Serre spectral sequences, and, in the case of a full product of groups, give Künneth formulas. The latter are used to make easy explicit computations of the homology and cohomology of an arbitrary finitely generated abelian group acting trivially on an arbitrary module.

We will prove the following:

Theorem 1. For $i=1,2$, let $f_{i}: B_{i} \rightarrow A$ be a surjection of groups with kernel $K_{i}$, and let $M_{i}$ be a left $B_{i}$-module. Let $B_{1} \times_{A} B_{2}$ $=\left\{\left(b_{1}, b_{2}\right) \in B_{1} \times B_{2} \mid f_{1}\left(b_{1}\right)=f_{2}\left(b_{2}\right)\right\}$, and consider $M_{1} \otimes_{z} M_{2}$ as a left $B_{1} \times_{A} B_{2}$-module in the natural fashion. If $N, N^{\prime}$ are left $A$-modules, let $Z A$ denote the integral group ring of $A$, and form $\operatorname{Tor}^{Z A}\left(N, N^{\prime}\right)$ by considering $N$ as a right $A$-module with $n a=a^{-1} n$. Then, if $\operatorname{Tor}_{1}^{Z}\left(M_{1}, M_{2}\right)=0$, there is a spectral sequence

$$
E_{p, q}^{2} \Rightarrow H_{n}\left(B_{1} \times_{A} B_{2}, M_{1} \otimes_{z} M_{2}\right)
$$

where

$$
E_{p, q}^{2}=\sum_{s+t=q} \operatorname{Tor}_{p}^{Z_{A}}\left(H_{s}\left(K_{1}, M_{1}\right), H_{t}\left(K_{2}, M_{2}\right)\right) .
$$

Again, consider $\operatorname{Hom}_{Z}\left(M_{1}, M_{2}\right)$ as a left $B_{1} \times_{A} B_{2}$-module with $\left(\left(b_{1}, b_{2}\right) f\right)(m)=b_{2} f\left(b_{1}^{-1} m\right)$. Then, if $\operatorname{Ext}_{Z}^{1}\left(M_{1}, M_{2}\right)=0$, there is a spectral sequence

$$
\begin{aligned}
\sum_{s+t=q} \operatorname{Ext}_{Z A}^{p}\left(H_{s}\left(K_{1}, M_{1}\right), H^{t}(\right. & \left.\left.K_{2}, M_{2}\right)\right) \\
\quad & \Rightarrow H^{n}\left(B_{1} \times_{A} B_{2}, \operatorname{Hom}_{Z}\left(M_{1}, M_{2}\right)\right) .
\end{aligned}
$$

Corollary. If $\operatorname{Tor}_{1}^{Z}\left(M_{1}, M_{2}\right)=0$, there are $Z$-split exact sequences

$$
\begin{aligned}
0 & \rightarrow \sum_{s+t=n} H_{s}\left(B_{1}, M_{1}\right) \otimes_{z} H_{t}\left(B_{2}, M_{2}\right) \rightarrow H_{n}\left(B_{1} \times B_{2}, M_{1} \otimes_{z} M_{2}\right) \\
& \rightarrow \sum_{s+t=n-1} \operatorname{Tor}_{1}^{z}\left(H_{s}\left(B_{1}, M_{1}\right), H_{t}\left(B_{2}, M_{2}\right)\right) \rightarrow 0,
\end{aligned}
$$

$a n d$, if $\operatorname{Ext}_{Z}^{1}\left(M_{1}, M_{2}\right)=0$,

Received by the editors July 3, 1969.

A MS Subject Classifications. Primary 1820, 2050.

Key Words and Phrases. Homology of groups, cohomology of groups, fiber product of groups, Hochschild-Serre spectral sequence, Künneth formula, homology of abelian groups, cohomology of abelian groups, universal coefficient theorems. 


$$
\begin{gathered}
0 \rightarrow \sum_{(1.4)} \operatorname{Ext}_{Z}^{1}\left(H_{s}\left(B_{1}, M_{1}\right), H^{t}\left(B_{2}, M_{2}\right)\right) \rightarrow H^{n}\left(B_{1} \times B_{2}, M_{1} \otimes_{Z} M_{2}\right) \\
\rightarrow \sum_{s+t=n} \operatorname{Hom}_{Z}\left(H_{8}\left(B_{1}, M_{1}\right), H^{t}\left(B_{2}, M_{2}\right)\right) \rightarrow 0 .
\end{gathered}
$$

Note that, for $B_{1}=A, M_{1}=Z,(1.1)$ and (1.2) become the Hochschild-Lyndon-Serre spectral sequences. If $M_{1}=Z, B_{2}=\{e\}$, (1.3) and (1.4) give the Universal Coefficient Theorems. When $B_{i}$ acts trivially on $M_{i},(1.3)$ follows from the Künneth formula and the Eilenberg-Zilber theorem in algebraic topology.

The sequences (1.3) and (1.4) give very easy explicit computations of the homology and cohomology of an arbitrary finitely generated abelian group acting trivially on an arbitrary module (cf. [3], [4]), and we close with these.

For the proof of Theorem 1, we will need the following generalizations to bifunctors of the spectral sequence associated to the composition of two functors.

Theorem 2. For $i=1,2$, let $F_{i}: \boldsymbol{C}_{\boldsymbol{i}} \rightarrow \boldsymbol{B}_{\boldsymbol{i}}$ be additive functors between abelian categories with enough projectives. Let $A$ be an abelian category, and let $G: B_{1} \times B_{2} \rightarrow A$ be additive in each variable. Suppose $L_{p} G\left(F_{1}\left(P_{1}\right), F_{2}\left(P_{2}\right)\right)=0$ whenever $P_{1}, P_{2}$ are projective and $p \geqq 1$. Then there is a spectral sequence

$$
\sum_{8+t=q} L_{p} G\left(L_{8} F_{1}\left(M_{1}\right), L_{t} F_{2}\left(M_{2}\right)\right) \Rightarrow L_{n}\left(G\left(F_{1} \times F_{2}\right)\right)\left(M_{1}, M_{2}\right) .
$$

Again, suppose that $A$ has enough projectives, that $C$ is abelian, and that $F: A \rightarrow C$ is additive. Then, if $L_{p} F\left(G\left(P_{1}, P_{2}\right)\right)=0$ whenever $P_{1}, P_{2}$ are projectives and $p \geqq 1$, there is a spectral sequence

$$
L_{p} F\left(L_{q} G\left(N_{1}, N_{2}\right)\right) \Rightarrow L_{n}(F G)\left(N_{1}, N_{2}\right) \text {. }
$$

Proof. The proof proceeds as in [2, Theorem 2.4.1]. Let us first consider, in the category $B_{1}$, a complex $U$ with the property that $B_{n}(U)$ and $Z_{n}(U)$ are direct summands of $U_{n}$, so that $U$ has the form:

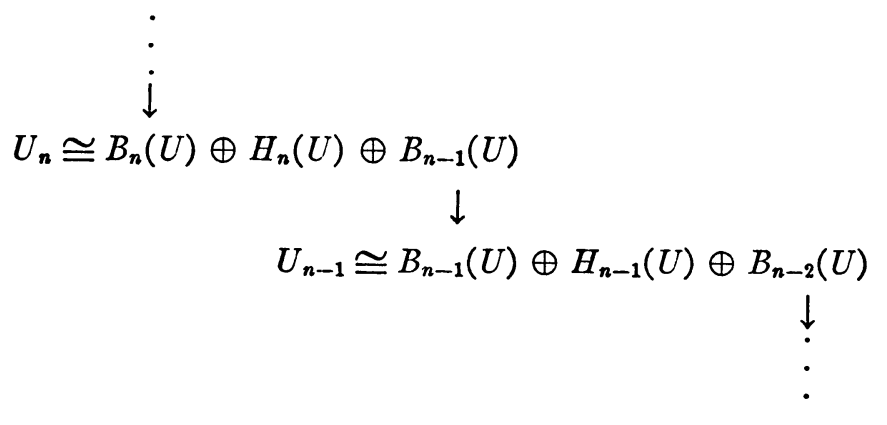


Let $U^{\prime}$ be another such complex in $B_{2}$. Consider $G\left(U, U^{\prime}\right)$, the complex associated to the double complex $\left\{G\left(U_{p}, U_{q}^{\prime}\right)\right\}$. This decomposes into the direct sum of nine complexes because of the decomposition of $U$ and $U^{\prime}$ into three as above. $H_{n} G\left(H(U), H\left(U^{\prime}\right)\right)$ $=\sum_{s+t=n} G\left(H_{s}(U), H_{t}\left(U^{\prime}\right)\right)$, and the other eight factors have trivial homology. Hence

$$
H_{n} G\left(U, U^{\prime}\right)=\sum_{s+l=n} G\left(H_{\imath}(U), H_{\iota}\left(U^{\prime}\right)\right)
$$

Now let $X$ be a projective resolution of $M_{1}$, and construct, as in [1, Chapter XVII], a double complex $Y$ such that, for each $p, q$, $B_{q}\left(Y_{p *}\right)$ and $Z_{q}\left(Y_{p *}\right)$ are direct summands of $Y_{p q}$, and, for each $q$, $Y_{* q}$ and $H_{q}^{l l}\left(Y_{* *}\right)$ are projective resolutions of $F_{1}\left(X_{q}\right)$ and $H_{q} F_{1}(X)$ $=L_{q} F_{1}\left(M_{1}\right)$ respectively. Construct $X^{\prime}, Y^{\prime}$ for $M_{2}$ similarly. Let $V_{p q}$ $=\sum_{s+t=p ; s^{\prime}+t^{\prime}=q} G\left(Y_{s s^{\prime}}, Y_{u^{\prime}}^{\prime}\right)$. Then

$$
H_{p}(V \cdot q)=\sum_{\bullet^{\prime}+t^{\prime}=q} I_{p} G\left(F_{1}\left(X_{\iota^{\prime}}\right), F_{2}\left(X_{\iota^{\prime}}^{\prime \prime}\right)\right)=0 \quad(p \geqq 1)
$$

and, by (2.3),

$$
H_{q}\left(V_{p^{*}}\right)=\sum_{s+l=p_{i} \star^{\prime}+t^{\prime}=q} G\left(H_{\boldsymbol{*}^{\prime}}\left(Y_{*^{*}}\right), H_{t^{\prime}}\left(Y_{\iota^{*}}\right)\right) .
$$

Hence one of the two spectral sequences associated to the double complex $V$ collapses to yield $H_{n}(V)=L_{n}\left(G\left(F_{1} \times F_{2}\right)\right)\left(M_{1}, M_{2}\right)$, and the other thus becomes (2.1).

To establish (2.2), let $X^{(i)}$ be a projective resolution of $N_{i}$, and let $Y$ be a double complex resolution (in the above sense) for the single complex associated to $\left\{G\left(X_{p}^{(1)}, X_{q}^{(2)}\right)\right\}$.

Proof of Theorem 1 . For any group $C, H_{n}(C,-)$ is the $n$th left derived functor of the functor $M \mapsto M_{C}$, where $M_{C}$ is the quotient of $M$ by the submodule generated by $\{\sigma m-m \mid m \in M, \sigma \in C\} \odot\left(Z B_{i}\right)_{K_{i}}$ $=Z A$, so that $M \mapsto M_{K_{i}}$ preserves projectives when considered as a functor to $A$-modules. Again, $Z B_{1} \otimes Z B_{2}=Z\left(B_{1} \times B_{2}\right)$, which is projective for the subgroup $B_{1} \times_{A} B_{2} \subset B_{1} \times B_{2}$ (consider a coset decomposition). Hence, since $M_{K_{1}} \otimes_{A} N_{K_{2}}=\left(M \otimes_{Z} N\right)_{B_{1} \times_{A} B_{2}}$, we obtain from Theorem 2 two spectral sequences with the same limit. The second of these collapses, because $\operatorname{Tor}_{1}^{Z}\left(M_{1}, M_{2}\right)=0$, to identify the limit. The first thus becomes (1.1).

$H^{n}(C,-)$ is the right derived functor of $M \mapsto M^{c}=\{m \in M \mid \sigma m=m$ for every $\sigma \in C\}$. Let $I$ be an injective $Z$-module. The right $C$-module structure of $Z C$ defines a left $C$-module structure for $\operatorname{Hom}_{Z}(Z C, I)$. The latter is injective, and every injective is a direct summand of one of this form. $\left(\operatorname{Hom}_{z}\left(Z B_{i}, I\right)\right)^{K_{i}} \cong \operatorname{Hom}_{Z}(Z A, I)$, so that $M \mapsto M^{K_{i}}$ 
preserves injectives. $\operatorname{Hom}_{Z A}\left(M_{K_{1}}, N^{R_{2}}\right)=\left(\operatorname{Hom}_{Z}(M, N)\right)^{B_{1} X_{A} B_{2}}$. Hence the result for cohomology follows analogously.

Proof of Corollary. When $A=\{e\}$ the spectral sequences (1.1) and (1.2) become the desired short exact sequences. It remains to show that the latter split. For $n=0$, this is trivial. Let $0 \rightarrow M^{1} \rightarrow P$ $\rightarrow M_{1} \rightarrow 0$ be exact with $P$ a projective $Z B_{1}$-module. We obtain isomorphisms $H_{n}\left(B_{1}, M_{1}\right) \simeq H_{n-1}\left(B_{1}, M^{1}\right)(n \geqq 2)$ and an exact sequence

$$
0 \rightarrow H_{1}\left(B_{1}, M_{1}\right) \underset{\beta}{\rightarrow} H_{0}\left(B_{1}, M^{1}\right) \underset{\alpha}{\rightarrow} H_{0}\left(B_{1}, P\right) .
$$

Now $H_{0}\left(B_{1}, P\right)=P_{B_{1}}$ is $Z$-projective. Hence so is image $\alpha$. Hence $\beta$ is split. From the exact sequence $0=\operatorname{Tor}_{1}^{Z}\left(M_{1}, M_{2}\right) \rightarrow M^{1} \otimes M_{2} \rightarrow P \otimes M_{2}$ $\rightarrow M_{1} \otimes M_{2} \rightarrow 0$ we also have a homomorphism $H_{n}\left(B_{1} \times B_{2}, M_{1} \otimes M_{2}\right)$ $\rightarrow H_{n-1}\left(B_{1} \times B_{2}, M^{1} \otimes M_{2}\right)$. The diagram

$$
\begin{gathered}
\sum_{l=0}^{n} H_{n-l}\left(B_{1}, M_{1}\right) \otimes H_{l}\left(B_{2}, M_{2}\right) \underset{\gamma}{\rightarrow} H_{n}\left(B_{1} \times B_{2}, M_{1} \otimes M_{2}\right) \\
\downarrow \\
\sum_{l=0}^{n-1} H_{n-l-1}\left(B_{1}, M^{1}\right) \otimes H_{l}\left(B_{2}, M_{2}\right) \underset{\gamma^{\prime}}{\rightarrow} H_{n-1}\left(B_{1} \times B_{2}, M^{1} \otimes M_{2}\right)
\end{gathered}
$$

commutes up to sign. (In fact, it comes from a morphism of double complexes of degree $(0,-1)$ : In the proof of Theorem 2 , choose $X$ such that $X_{0}=P$, and let $X_{q}^{\prime \prime}=X_{q+1}$, so that $X^{\prime \prime}$ is a projective resolution of $M^{1}$.) We may assume by induction that $\gamma^{\prime}$ is split, and this allows us to find $\delta: H_{n}\left(B_{1} \times B_{2}, M_{1} \otimes M_{2}\right) \rightarrow \sum_{l=0}^{n-1} H_{n-l}\left(B_{1}, M_{1}\right)$ $\otimes H_{l}\left(B_{2}, M_{2}\right)$ which splits the restriction of $\gamma$. Moreover, $H_{0}\left(B_{1}, M_{1}\right)$ $\otimes H_{n}\left(B_{2}, M_{2}\right) \rightarrow H_{n}\left(B_{1} \times B_{2}, M_{1} \otimes M_{2}\right)$, when followed by $\delta$, is zero. The inductive assumption also allows us to find $\eta^{\prime}: H_{n}\left(B_{1} \times B_{2}, M_{1} \otimes M_{2}\right)$ $\rightarrow H_{n}\left(B_{1}, M_{1}\right) \otimes H_{0}\left(B_{2}, M_{2}\right)$ which splits the restriction of $\gamma$ and which gives zero when preceded by $\sum_{l=1}^{n} H_{n-l}\left(B_{1}, M_{1}\right) \otimes H_{l}\left(B_{2}, M_{2}\right)$ $\rightarrow H_{n}\left(B_{1} \times B_{2}, M_{1} \otimes M_{2}\right)$. By symmetry, there is $\eta: H_{n}\left(B_{1} \times B_{2}, M_{1} \otimes M_{2}\right)$ $\rightarrow H_{0}\left(B_{1}, M_{1}\right) \otimes H_{n}\left(B_{2}, M_{2}\right)$ which, together with $\delta$, defines a splitting for $\gamma$.

Similarly for cohomology.

Computations for finitely generated abelian groups. Let $Z_{a}$ be the group of integers modulo $a$, and suppose $a_{m}\left|a_{m-1}\right| \cdots \mid a_{1}$. One shows, for $n \geqq 1$,

$$
H_{n}\left(Z_{a_{1}} \times \cdots \times Z_{a_{m}}, Z\right)=\sum_{s=1}^{m} \phi(s, n) Z_{a_{s}}
$$

where $\phi(s, n)=\sum_{l=1}^{n} \phi(s-1, t)(s>1), \phi(1, n)=1$ if $n$ is odd, 0 otherwise, $l Z_{a_{s}}=Z_{a_{s}} \oplus \cdots \oplus Z_{a_{s}}(l$ times). (The module structure for $Z$ is 
the trivial one.) The proof is by induction on $m$, starting from the cyclic group case $(m=1)$. Apply (1.3) to $Z_{a_{1}} \times\left(Z_{a_{2}} \times \cdots \times Z_{a_{m}}\right)$. Similarly, if $Z^{l}=Z \times \cdots \times Z$ ( $l$ times $)$,

$$
H_{n}\left(Z^{l}, Z\right)=\left(\begin{array}{l}
l \\
n
\end{array}\right) Z \text {. }
$$

Now consider an arbitrary finitely generated abelian group $G=Z_{a_{1}} \times \cdots \times Z_{a_{m}} \times Z^{l}$. Using (1.3), we may combine the two previous results to obtain

$$
H_{n}(G, Z)=\sum_{s=0}^{m} \phi(s, l, n) Z_{a}
$$

where

$$
a_{0}=0, \quad \phi(s, l, n)=\sum_{l=1}^{n}\left(\begin{array}{c}
l \\
n-t
\end{array}\right) \phi(s, t)(s, n \geqq 1), \quad \phi(0, l, n)=\left(\begin{array}{l}
l \\
n
\end{array}\right),
$$

and $\phi(s, l, 0)=0(s \geqq 1)$.

Finally, if $G$ acts trivially on an arbitrary abelian group $M$, (1.3) yields $H_{n}(G \times\{e\}, Z \otimes M)=H_{n}(G, Z) \otimes M \oplus \operatorname{Tor}_{1}^{Z}\left(H_{n-1}(G, Z), M\right)$, whence

$$
H_{n}(G, M)=\sum_{s=0}^{m} \phi(s, l, n) M_{a_{s}} \oplus \sum_{s=1}^{m} \phi(s, l, n-1)_{a_{s}} M,
$$

where $M_{a_{s}}=M / a_{s} M,{ }_{a} M=\left\{m \in M \mid a_{s} m=0\right\}$. Similarly, using (1.4),

$$
H^{n}(G, M)=\sum_{s=0}^{m} \phi(s, l, n)_{a_{s}} M \oplus \sum_{s=1}^{m} \phi(s, l, n-1) M_{a_{s}} .
$$

\section{BibLIOGRAPHY}

1. H. Cartan and S. Eilenberg, Homological algebra, Princeton Univ. Press, Princeton, N. J., 1956. MR 17, 1040.

2. A. Grothendieck, Éléments de gêmêtrie algêbrique. IV: Études locale de schémas et des morphismes de schémas. I, Inst. Hautes Études Sci. Publ. Math. No. 20 (1964). MR $30 \# 3885$.

3. R. C. Lyndon, The cohomology theory of group extensions, Duke Math. J. 15 (1948), 271-292. MR 10, 10.

4. B. Pareigis, Zur Kohomologie endlich erzeugter abelscher Gruppen, Bayer. Akad. Wiss. Math.-Natur. Kl. S.-B. 1967, Abt. II, 177-193.

Institut de Recherche Mathematique Avancee, Strasbourg 